\title{
BIÓPSIA DO NERVO SURAL NA DISTROFIA MUSCULAR MIOTÔNICA
}

\author{
GABRIEL R. DE FREITAS*, MARCOS R.G. DE FREITAS* *, OSVALDO J.M. NASCIMENTO** *
}

\begin{abstract}
RESUMO - Foram estudados 12 pacientes com distrofia muscular miotônica com o objetivo de verificar o comprometimento do sistema nervoso periférico. Todos apresentavam os sinais e sintomas principais da doença. Nenhum tinha outras causas que pudessem justificar uma polineuropatia. Todos foram submetidos a biópsia do nervo sural com contagem de fibras mielínicas e realização de histograma. Dois não apresentavam histograma bimodal. Os pacientes mostraram redução do número de fibras mielínicas. Concluímos que a polineuropatia pode fazer parte do quadro clínico polimorfo da distrofia muscular miotônica.
\end{abstract}

PALAVRAS-CHAVE: distrofia muscular miotônica, sistema nervoso periférico, polineuropatia, nervo sural, biópsia.

\section{Sural nerve biopsy in myotonic dystrophy}

ABSTRACT - Twelve patients with myotonic dystrophy were studied to look for the involvement of the peripheral nervous system in this disease. All of them showed the main signs and symptoms of the disease. They did not have another causes to justify a polineuropathy. They were submitted to sural nerve biopsy with counting of myelinated fibers and histogram. Patients showed a reduction in the number of myelinated fibers and in two patients the histogram was unimodal. We concluded that polineuropathy may be another multisystemic manifestation of myotonic dystrophy.

KEY WORDS: myotonic dystrophy, peripheral nervous system, polineuropathy, sural nerve, biopsy.

Distrofias musculares são definidas classicamente como doenças dos músculos esqueléticos degenerativas, progressivas e hereditárias' ${ }^{1}$. A distrofia muscular miotônica (DMM) é a miopatia degenerativa mais comum em adultos. Apresenta incidência de 13,5 por 100000 indivíduos nascidos vivos e prevalência entre 2,4 e 5,5 por 100000 habitantes $^{9}$. Foi descrita pela primeira vez em 1909 por Steinert e, no mesmo ano, por Batten e Gibb (apud Adams e Victor ${ }^{1}$ ). É doença transmitida de modo autossômico dominante e afeta vários sistemas e orgãos. Caracteriza-se por miotonia, fraqueza muscular, amiotrofia e numerosas manifestaçōes não musculares, como calvície frontal, catarata, atrofia testicular, anormalidades da condução cardíaca, insuficiência respiratória, distúrbios do aparelho gastrointestinal e hipersonia. A atrofia tem predileção por certos grupos musculares: face, pescoço e extremidades dos membros. Tal predileção confere à facies um aspecto magro, alongado, inexpressivo com ptose palpebral bilateral e boca entreaberta: facies "em machadinha". A miotonia (incapacidade de relaxamento muscular imediato) é melhor demonstrada na mão e língua, e diminui com a progressão da doença. Recentemente foi descoberto que na DMM há repetição anormal de

Serviço de Neurologia da Faculdade de Medicina da Universidade Federal Fluminense (UFF): *Interno; **Professor Titular, Chefe do Serviço; ***Professor Titular. Aceite: 1-setembro-1995. 
um trinucleotídeo no braço longo do cromossoma 192.10. A repetição do trinucleotídeo CTG é transcrita num RNA mensageiro. Esse RNA possui um polipeptídeo semelhante à família da proteína quinase, daí ser denominado miotonina proteína quinase. Porém, nāo se sabe ainda como a miotonina proteína quinase participa da fisiopatogenia da doença. $O$ tamanho da repetição está diretamente relacionado à gravidade e ao início da doença.

Várias evidências, clínicas e eletrofisiológicas ${ }^{7.12}$, de polineuropatia na DMM foram relatadas. Até o sistema nervoso autônomo já foi referido como acometido ${ }^{16}$. Estudos patológicos do nervo periféríco sāo escassos na literatura e, muitas vezes, divergentes ${ }^{4.13}$. Nosso objetivo, neste estudo é a análise do nervo sural em pacientes com DMM a fim de verificar o comprometimento do sistema nervoso periférico (SNP) nesta doença.

\section{PACIENTES E MÉTODOS}

Estudamos a biópsia do nervo sural em 12 pacientes com DMM. Eram oito homens e quatro mulheres, com a idade mínima de 14 anos e a máxima de 54 anos. Todos foram vistos no Setor de Doenças Neuromusculares do Serviço de Neurologia do Hospital Universitário Antônio Pedro da UFF. Foram avaliados clinicamente, submetidos à eletromiografia e em nove dos pacientes foi estudada a neurocondução. Na Tabela l, encontramse as características clínicas desses pacientes. Nenhum deles apresentava história de alcoolismo ou uso de outras substâncias tóxicas, desnutrição, diabete melito ou outras doenças metabólicas que podem levar a neuropatia periférica. Os Pacientes 3 e 4 eram irmãos, assim como os Pacientes 9 e 10.

Na eletromiografia, em todos observou-se a presença de fenômeno miotônico nos músculos proximais e distais, potenciais polifásicos de baixa amplitude, e recrutamento completo aos mínimos esforços. A Tabela 2 mostra os principais resultados da neurocondução sensitivo-motora.

O nervo sural foi retirado em sala cirúrgica e fixado em glutaraldeído. Cortes semi-finos transversais e longitudinais $(\mathrm{I} \mu \mathrm{m})$ foram corados com azul de toluidina para a microscopia óptica. Determinou-se a densidade e o diâmetro das fibras mielínicas (FM) para realização de histograma em todos os casos.

Tabela 1: Quadro clínico dos pacientes.

Paciente Iniciais Idade Sexo Cor Força Atrofia Miotonia Sensibilidade Alopécia Hereditariedade distal muscular distal

\begin{tabular}{ccccccccccc}
\hline I & CBS & 30 & F & B & ++ & P & P & N & Não & D \\
2 & JEF & 46 & M & B & N & P & P & N & Sim & D \\
3 & FABB & 20 & M & B & N & P & P & N & Não & Sim \\
4 & FBB & 14 & M & B & N & P & P & N & Não & Sim \\
5 & ERPS & 46 & F & B & + & P & P & N & Não & Sim \\
6 & AOCN & 26 & M & B & ++ & P & P & N & Não & Sim \\
7 & SAC & 44 & M & B & + & P & P & N & Não & Sim \\
8 & SMA & 35 & F & B & ++ & P & A & + & Não & Sim \\
9 & TMFS & 29 & F & B & ++ & P & P & N & Não & Sim \\
10 & TAF & 28 & M & B & + & P & P & N & Não & Sim \\
11 & LCN & 54 & M & B & +++ & P & P & N & Sim & Sim \\
12 & RRS & 46 & M & B ++ & P & P & + & Sim & Sim \\
\hline
\end{tabular}

B, Branca; N, Normal; P, Presente; A, Ausente; D, Desconhecida; +++ , Gravemente alterado); ++ , Moderadamente alterado; + , Levemente alterado. 
Tabela 2 . Neuroconduçäo dos pacientes.

\begin{tabular}{|c|c|c|c|c|c|c|c|c|c|c|c|c|c|c|c|c|c|c|}
\hline \multirow{3}{*}{ P. } & \multicolumn{9}{|c|}{ Motora } & \multicolumn{9}{|c|}{ Sensitiva } \\
\hline & \multicolumn{3}{|c|}{ Mediano } & \multicolumn{3}{|c|}{ Ulnar } & \multicolumn{3}{|c|}{ Fibular } & \multicolumn{3}{|c|}{ Mediano } & \multicolumn{3}{|c|}{ Ulnar } & \multicolumn{3}{|c|}{ Sural } \\
\hline & VCN & LD & A & VCN & LD & A & $\mathrm{VCN}$ & $\mathrm{LD}$ & A & VCN & LD & a & $\mathrm{VCN}$ & LD & $\mathbf{a}$ & $\mathrm{VCN}$ & LD & $\mathbf{a}$ \\
\hline 2 & 53,7 & 3,8 & NR & 53 & 3,7 & NR & 42 & 7,6 & NR & NR & 4,6 & NR & NR & 5 & NR & NR & 5,5 & NR \\
\hline 3 & 77 & 3,3 & 10 & 49 & 2,7 & 11 & 44 & 4,2 & 4 & 46 & 2,8 & 42 & 45 & 2,9 & 30 & 38 & 3,2 & 28 \\
\hline 4 & 61 & 3,3 & 8 & 59 & 2,5 & 8 & 47 & 3,1 & 5 & 48 & 2,6 & 20 & 52 & 2,5 & 20 & 42 & 2,4 & 10 \\
\hline 5 & 49 & 5,0 & NR & 48 & 4,8 & NR & 47,6 & 3,7 & NR & 50 & 3,1 & NR & 51 & 3,5 & NR & NR & 3,9 & NR \\
\hline 7 & 45,5 & 5,5 & NR & 40 & 5,0 & NR & 43 & 6,0 & NR & 50 & 4,3 & NR & 49 & 4,0 & NR & NR & 5,6 & NR \\
\hline 8 & 52 & 3,2 & NR & 51 & 3,1 & NR & 50 & 3,3 & 6 & 54 & 3,4 & NR & 55 & 3,4 & NR & NR & 3,6 & NR \\
\hline 9 & 54 & 3,1 & NR & 59 & 3,2 & NR & 49 & 3,2 & NR & 57 & 3,0 & 58 & 3,2 & NR & NR & NR & 3,5 & NR \\
\hline 11 & 54 & 2,8 & 2,5 & 55 & 2,6 & 10 & 40 & 6 & 0,1 & 54 & 2,4 & 20 & 54 & 2,4 & 18 & 0 & 0 & 0 \\
\hline 12 & 60 & 3,1 & NR & $63, \mathrm{I}$ & 3,2 & NR & NR & NR & NR & NR & 3,2 & NR & NR & 3,4 & NR & NR & NR & NR \\
\hline
\end{tabular}

P., Paciente; VCN, velocidade de condução nervosa ( $\mathrm{m} / \mathrm{seg}) ; L D$, latência distal ( $\mathrm{m} / \mathrm{seg}) ; A$, amplitude ( $\mathrm{mV}$ ); a. amplitude (uv); $N R$, não realizado

\section{RESULTADOS}

Dos nove pacientes submetidos à neurocondução sensitivo-motora, cinco apresentaram alteração sugerindo comprometimento axonal (Casos 2,5,7,8, 11). Em um nāo houve resposta no nervo sural (Caso 11), em quatro a latência distal estava aumentada (Casos $2,5,7,8$ ) e em três as latências distais motoras estavam aumentadas (Casos 2,5,7). A amplitude do potencial de ação

Tabela 3. Contagem de fibras mielinicas $(F M)$ dos pacientes.

\begin{tabular}{cc}
\hline Paciente & Número de FM/mm ${ }^{2}$ \\
\hline 1 & 6587 \\
3 & 2618 \\
4 & 9618 \\
5 & 10618 \\
6 & 5443 \\
7 & 7082 \\
8 & 8288 \\
9 & 6690 \\
10 & 6773 \\
11 & 7989 \\
12 & 5340 \\
\hline
\end{tabular}
sensitivo e motor não foi determinada em todos os pacientes; mostrou-se reduzida em um enfermo (Caso 11).

A contagem de FM dos pacientes com DMM revelou perda de fibras. $O$ número de FM por milímetro quadrado variou entre 2618 e 10618 (média 7043 FM/mm²) (Tabela 3).

O histograma de dez pacientes com DMM não mostrou anormalidades no número de picos, apresentando padrão bimodal, com um pico entre fibras de $3 \mathrm{e}$ 6 micra de diâmetro e outro entre fibras de 9 a 13 micra de diâmetro (Figura 1). Entretanto dois pacientes revelaram histogramas anormais: um unimodal (Caso 11) e outro sem nenhum pico (Caso 2). O Caso 2 mostrou número extremamente reduzido de fibras para a sua idade (Fig 2). 
Caso 4

F.B.B.

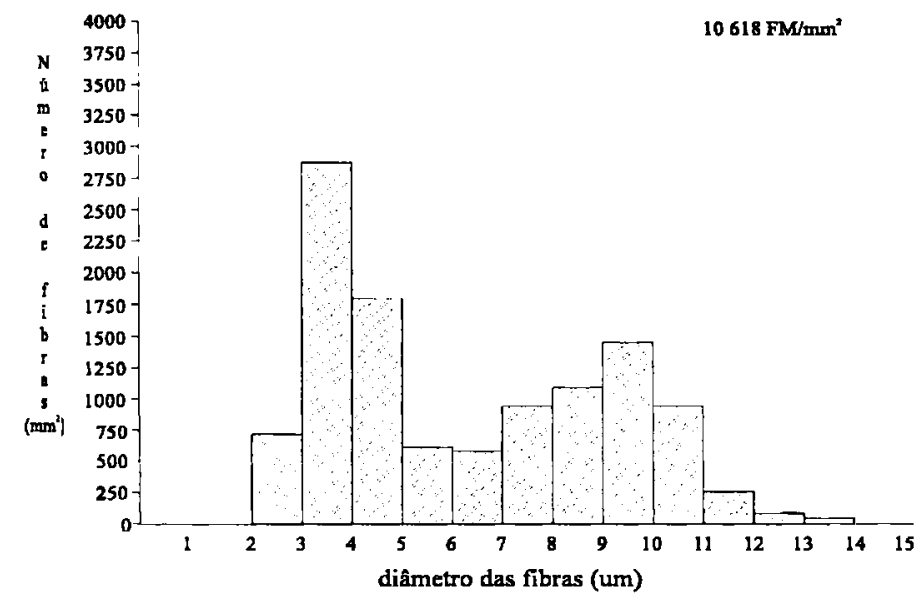

Figura 1. Histograma: caso 4. Padrão bimodal, número de fibras mielínicas normal para sua idade.

Caso 2

J.E.F.

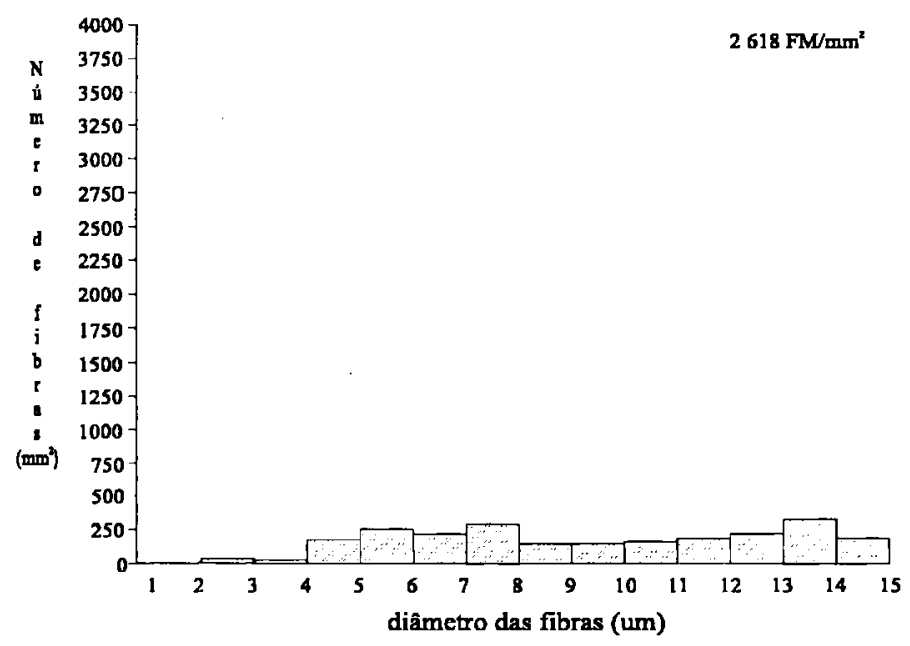

Figura 2. Histograma: caso 2. Número de fibras mielínicas bem inferior ao normal para sua idade.Não apresenta pico. 


\section{DISCUSSÃO}

Embora a DMM tenha sido descrita pela primeira vez no início deste século e manifestações de polineuropatia venham sendo relatadas há algum tempo, são relativamente recentes e escassos os estudos que objetivam mostrar as alterações patológicas no SNP. Existe número bem maior de trabalhos estudando a miopatia, alterações cardíacas, oculares e digestivas provocadas pela doença.

Pela neurocondução verificamos que dos nove pacientes que realizaram o exame, cinco tiveram quadro de polineuropatia do tipo axonal. Mondelli c col. ${ }^{12}$ fizeram estudo neurofisiológico de 24 pacientes que apresentavam DMM. Em 11 deles (46\%) encontraram alterações compatíveis com polineuropatia axonal, acometendo tanto fibras sensitivas quanto motoras.

Nosso estudo mostrou que os pacientes com DMM, além de distúrbios clínicos e eletrofisiológicos do SNP, apresentava alterações na biópsia do nervo sural. Quando comparados com os histogramas do nervo sural de seis indivíduos sãos e com neurocondução normal obtidos por Dyck e col. ${ }^{5}$ pelos mesmos métodos, cinco dos nossos pacientes $(42 \%)$ revelaram diminuiçāo significativa do número de FM. Estudo semelhante foi realizado por Cross e col. ${ }^{4}$, que mostrou perda leve de FM em 11 de 13 pacientes (85\%) com DMM escolhidos aleatoriamente.

Entretanto, nossos achados apresentam discrepância com os resultados obtidos por Pollock e Dyck ${ }^{13}$. Esses autores cstudaram quatro pacientes com DMM e não encontraram reduçāo significativa do número de FM, achando que não havia alterações patológicas no SNP na DMM. Tal fato pode ser devido a dois motivos. Pollock e Dyck estudaram nervos mais proximais do que o sural como o nervo fibular superficial e o fascículo lateral do nervo do fibular profundo, nervos estes menos afetados nas polineuropatias. Pensamos porém que o principal motivo foi que esses autores afastaram os pacientes com alteraçōes de neuroconduçāo e selecionaram somente aqueles com conduçāo nervosa normal.

Não evidenciamos correlação entre a polineuropatia e a gravidade da miopatia. Talvez isso possa ser explicado pelo fato da polineuropatia ser mais um evento da DMM, assim como o são as outras manifestações da doença, como por exemplo a atrofia testicular, as alterações da condução cardíaca, que podem estar presentes ou faltar em alguns casos. A polineuropatia ocasionalmente pode ser a manifestaçāo predominante da docnça ${ }^{14}$ ou preceder a miopatia. De fato, a situaçāo da neuropatia pode ser semelhante à do comprometimento cardíaco. Um estudo mostrou famílias de pacientes com DMM com alterações cardíacas e outras familias que não apresentavam alterações do coração".

Entretanto, nosso estudo e outros na literatura ${ }^{4,7.12}$ mostram que a polineuropatia é evento frequente na DMM e talvez seja amiúde nāo diagnosticada por ser mascarada pela característica maior da doença, a miopatia distal.

A gravidade do acometimento de alguns orgãos na DMM parece ser diretamente proporcional ao tamanho da repetição de trinucleotídeos $\mathrm{CTG}^{\mathrm{R}, 11}$. Estudo realizado por Jaspert e col. ${ }^{8}$ mostrou maior frequência de acometimento mental e gonadal nos pacientes com maior repetição, porém não houve correlação do tamanho da repetição com catarata, anormalidades gastrointestinais ou cardíacas. Entretanto, os resultados dos estudos acerca da repetição de trinucleotídeos são divergentes e Turnpenny e col. ${ }^{15}$ revelaram, utilizando 55 pacientes, que o grau de acometimento mental nāo se correlaciona com o número de trinucleotideos. Não existem estudos na literatura correlacionando a neuropatia com o tamanho da repetiçāo.

A natureza da polineuropatia permanece desconhecida. Mais estudos sobre o papel da repetição do trinucleotídeo CTG certamente irão mostrar o mecanismo fisiopatológico da polineuropatia e das outras manifestações da DMM.

Agradecimentos - Gostaríamos de agradecer a colaboração da Professora Maria Eugênia Duarte pelo empréstimo do equipamento necessário a contagem de fibras, a Guilherme Moraes de Azeredo e Marcela Rodríguez de Freitas pela análise e entrada dos dados no computador. 


\section{REFERÊNCIAS}

1. Adams RD, Victor M. Principles of neurology. Ed 4. New York: McGraw-Hill, 1989: 1117-1132. (The muscular dystrophies).

2. Brook JD, McCurrach ME, Harley HG et al. Molecular basis of myotonic dystrophy: expansion of a trinucleotide (CTG) repeat at the 3'end of a transcript encoding a protein kinase family member. Cell 1992 , 68:799-808.

3. Brunner HG, Spaans F, Smeets HJM, Coerwinkel-Driessen M, Hulsebos T, Wieringa B, Ropers HH. Genetic linkage with chromosome 19 but not chromosome 17 in a family with myotonic dystrophy associated with hereditary motor and sensory neuropathy. Neurology 1991, 41:80-84.

4. Cros D, Handen P, Pouget J, Pellisier JF, Gastaut JL, Serratrice G. Peripheral neuropathy in myotonic dystrophy: a nerve biopsy study. Ann Neurol 1988, 23:470-476.

5. Dyck PJ, Giannini C, Lais A. Pathological alterations of nerves. In Dyck PJ, Thomas PK (eds). Peripheral neuropathy. Ed 3. Philadelphia: Saunders, 1993; 514-595.

6. Hawley RJ, Gottdiener JS, Gay JA, Engel WK. Families with myotonic dystrophy with and without cardiac involvement. Arch Intern Med 1983, 143:2134-2135.

7. Jamal GA, Weir AI, Hansen S, Ballantyne JP. Myotonic dystrophy: a reassessment by conventional and more recently introduced neurophysiological techniques. Brain 1986, 109:1279-1296.

8. Jaspert A, Fahsold R, Grehl H, Claus D. Myotonic dystrophy: correlation of clinical symptoms with the size of the CTG trinucleotide repeat. J Neurol 1995, 242:99-104.

9. Jozefowicz RF, Griggs RC. Myotonic dystrophy. Neurol Clin 1988, 6:455-472.

10. La Spada AR, Baulson HL, Fischbeck KH. Trinucleotide repeat expansion in neurological diseases. Ann Neurol 1994, 36:814-822.

11. Mastrogiacomo I, Pagani E, Novelli G, Angelini C, Menegazzo E, Bonanni G, Dallapiccola B. Male hypogonadism in myotonic dystrophy is related to (CTG)n triplet mutation. J Endocrinol Invest 1994, 17:38L-383.

12. Mondelli M, Rossi A, Malandrini A, Della Porta P, Guazzi GC. Axonal motor and sensory neuropathy in myotonic dystrophy. Acta Neurol Scand 1993, 88:141-148.

13. Pollock M, Dyck PJ. Peripheral nerve morphometry in myotonic dystrophy. Arch Neurol 1976, 33:33-39.

14. Von Giesen HJ, Stoll G, Koch MC, Benecke R. Mixed axonal-demyelinating polyneuropathy as predominant manifestation of myotonic dystrophy. Muscle Nerve 1994, 17:701-703.

15. Tumpenny $P$, Clark C, Kelly K. Intelligence quotient profile in myotonic dystrophy, intergenerational deficit, and correlation with CTG amplification. J Med Gen 1994, 31:300-305.

16. Yoshida MM, Krishnamurthy S, Wattchow DA, Furness JB, Schuffler MD. Megacolon in myotonic dystrophy caused by a degenerative neuropathy of the myenteric plexus. Gastroenterology 1988, 95:820-827. 